\title{
Von Spondyloarthritis bis Osteoporose - Beteiligung des Skelettsystems bei chronisch entzündlichen Darmerkrankungen
}

\author{
Schüle, Solvey ; Frey, Diana ; Biedermann, Luc ; Grueber, Maude Martinho ; Zeitz, Jonas ; Vavricka, Stephan ;
} Möller, Burkhard ; Rogler, Gerhard ; Misselwitz, Benjamin

\begin{abstract}
Zusammenfassung. Bei chronisch entzündlichen Darmerkrankungen (Inflammatory Bowel Diseases, IBD) können nicht-entzündliche Gelenkschmerzen und entzündliche Gelenkmanifestationen auftreten. Letztere gehören zur Gruppe der Spondyloarthritiden. Sie werden am Achsenskelett als entzündlicher Rückenschmerz mit nächtlichen Schmerzen, Morgensteifigkeit und Besserung unter Aktivität manifest. Einschränkungen der Gelenkfunktion sind ebenfalls möglich. Bei anderen Patienten stehen periphere Gelenkbeschwerden im Vordergrund. Als Schmerzmedikamente werden COX-2-selektive, nicht-steroidale Antirheumatika (NSAR) empfohlen, da unselektive NSAR die zugrundeliegende IBD verschlimmern können. Am Achsenskelett werden Physiotherapie und Tumornekrosefaktor(TNF)-Inhibitoren eingesetzt, während bei peripherem Gelenkbefall Steroidinjektionen, Sulfasalazin und TNF-Inhibitoren wirksam sind. Entzündung, Malabsorption und Steroide führen bei IBDPatienten zu Osteopenie und Osteoporose. Bei langdauernder Krankheitsaktivität bzw. langer Steroidgabe ist ein Screening mit DXA-Scan indiziert. Therapeutisch sollten ausreichend Kalzium und Vitamin D sowie gegebenenfalls Bisphosphonate gegeben werden. Abstract. Inflammatory bowel diseases (IBD) are frequently accompanied by non-inflammatory joint pain and inflammatory spondyloarthritides. Spondyloarthritides can restrict joint function and typically manifest with inflammatory back pain with nightly pain and morning stiffness that improves upon exercising. In other patients, small or large peripheral joints are predominantly involved. Treatment comprises pain medication including COX-II selective non-steroidal anti-inflammatory drugs (NSAID), since nonselective NSAID can aggravate IBD. For axial manifestations, physiotherapy and tumor necrosis factor (TNF) inhibitors are effective, while for peripheral manifestations steroid injections, sulfasalazine and TNF inhibitors are useful. Osteopenia and osteoporosis may result from inflammation, malabsorption and/or steroids. Long-lasting disease activity or steroid treatment should prompt osteoporosis screening. Adequate calcium and vitamin D intake must be ensured and treatment with bisphosphonates evaluated. Résumé. Les maladies inflammatoires intestinales chroniques peuvent être associées à des arthralgies non inflammatoires et inflammatoires, ces dernières appartenant aux en miniscule spondylarthropathies. L'atteinte axiale comprend des dorsalgies nocturnes et une raideur matinale améliorée par le mouvement. Chez certains patients, les arthropathies périphériques prédominent. Les anti-inflammatoires non stéroïdiens COX-II sélectifs sont la base du traitement. L'atteinte axiale est traitée efficacement par la physiothérapie et les anti-TNF, alors que les atteintes périphériques sont traitées par la en miniscule sulfasalazine, les injections de stéroïdes ou les anti-TNF. L'inflammation, la malabsorption et les stéroïdes précipitent une ostéopénie/ostéoporose. La densitométrie osseuse est indiquée en cas d'inflammation prolongée ou de prise de stéroïdes. Un apport suffisant de calcium et vitamine D est essentiel. Un traitement par biphosphonates est parfois nécessaire.
\end{abstract}

DOI: https://doi.org/10.1024/1661-8157/a003301

Other titles: From Axial Spondyloarthritis to Osteoporosis - Spectrum of Skeletal Involvement in Inflammatory Bowel Diseases 
ZORA URL: https://doi.org/10.5167/uzh-178715

Journal Article

Accepted Version

Originally published at:

Schüle, Solvey; Frey, Diana; Biedermann, Luc; Grueber, Maude Martinho; Zeitz, Jonas; Vavricka, Stephan; Möller, Burkhard; Rogler, Gerhard; Misselwitz, Benjamin (2019). Von Spondyloarthritis bis Osteoporose - Beteiligung des Skelettsystems bei chronisch entzündlichen Darmerkrankungen. Praxis, 108(12):799-806.

DOI: https://doi.org/10.1024/1661-8157/a003301 


\title{
Von Spondyloarthritis bis Osteoporose - Beteiligung des Skelettsystems bei chronisch entzündlichen Darmerkrankungen
}

\author{
From Axial Spondyloarthritis to Osteoporosis - Spectrum of \\ Skeletal Involvement in Inflammatory Bowel Diseases
}

\section{De la Spondylarthrite à l'Ostéoporose - Spectre des atteintes squelettiques des Maladies inflammatoires intestinales chroniques}

Solvey Schüle ${ }^{1)}$, Diana Frey2), Luc Biedermann"1), Maude Martinho Grueber ${ }^{3)}$, Jonas Zeitz $^{1), 4)}$, Stephan Vavricka ${ }^{1)}$, Burkhard Möller ${ }^{5}$, Gerhard Rogler ${ }^{1)}$, Benjamin Misselwitz ${ }^{1,3)}$

1) Klinik für Gastroenterologie und Hepatologie, Universitätsspital Zürich und Universität Zürich, Rämistrasse 100, 8091 Zürich

2) Klinik für Rheumatologie, Universitätsspital Zürich und Universität Zürich, Gloriastrasse 25, 8091 Zürich

3) Universitätsklinik für Viszerale Chirurgie und Medizin, Inselspital Bern, Freiburgstrasse 18, 3010 Bern

4) GastroZentrum Hirslanden, Klinik Hirslanden, Witellikerstrasse 40, 8008 Zürich

5) Universitätsklinik für Rheumatologie, Immunologie und Allergologie, Inselspital Bern, Freiburgstrasse 10, 3010 Bern

\section{Abkürzungen}

\begin{tabular}{|l|l|}
\hline AS & Ankylosierende Spondylitis \\
\hline DXA & Dual-Energy X-Ray Absorptiometrie \\
\hline EIM & Extraintestinale Manifestationen \\
\hline HIV & Humanes Immundefizienz-Virus \\
\hline HLA & Humane Leukozytenantigene \\
\hline IBD & Inflammatory bowel disease, chronisch entzündliche Darmerkrankungen \\
\hline NSAR & Nicht-steroidale Antirheumatika \\
\hline SpA & Axiale Spondyloarthritis \\
\hline TNF & Tumornekrosefaktor \\
\hline
\end{tabular}




\section{Zusammenfassungen}

Bei chronisch entzündlichen Darmerkrankungen (englisch IBD) können nicht-entzündliche Gelenkschmerzen und entzündliche Gelenkmanifestationen auftreten. Letztere gehören zur Gruppe der Spondyloarthritiden. Sie werden am Achsenskelett als entzündlicher Rückenschmerz mit nächtlichen Schmerzen, Morgensteifigkeit und Besserung unter Aktivität manifest. Einschränkungen der Gelenkfunktion sind ebenfalls möglich. Bei anderen Patienten stehen periphere Gelenkbeschwerden im Vordergrund. Als Schmerzmedikamente werden COX-II selektive nicht-steroidale Antirheumatika (NSAR) empfohlen, da unselektive NSAR die zugrundeliegende IBD verschlimmern können. Am Achsenskelett werden Physiotherapie und Tumornekrosefaktor-(TNF)-Inhibitoren eingesetzt, während bei peripherem Gelenkbefall Steroidinjektionen, Sulfasalazin und TNFInhibitoren wirksam sind.

Entzündung, Malabsorption und Steroide führen bei IBD-Patienten zu Osteopenie und Osteoporose. Bei langdauernder Krankheitsaktivität bzw. langer Steroidgabe ist ein Screening mit DXA Scan indiziert. Therapeutisch sollten ausreichend Kalzium und Vitamin D sowie gegebenenfalls Bisphosphonate gegeben werden.

\section{Schlüsselwörter}

Chronisch entzündliche Darmerkrankungen, extraintestinale Manifestationen, axiale Spondyloarthritis, Osteoporose, Osteopenie, Arthralgie 


\section{Extraintestinale Manifestationen bei chronisch entzündlichen Darmerkrankungen}

Chronisch entzündliche Darmerkrankungen (englisch inflammatory bowel disease, IBD) umfassen chronisch rezidivierende Darmentzündungen mit den wichtigsten Krankheiten Morbus Crohn und Colitis ulcerosa. Bei beiden Erkrankungen wird der klinische Verlauf nicht allein durch die Darmentzündung, sondern in vielen Fällen auch durch extraintestinale Symptome bestimmt (Tabelle 1). Man unterscheidet dabei extraintestinale Manifestationen (EIM) der IBD, bei denen ein Organ-Befall ausserhalb des Darmes vorliegt (wie z.B. Gelenkschmerzen, Gelenksentzündungen, Uveitis, primär sklerosierende Cholangitis, Pyoderma gangränosum, Erythema nodosum usw.), von extraintestinalen Komplikationen, die Folgen der Entzündung darstellen (wie z.B. Gallensteine und Nierensteine [1], Anämie durch Eisen- oder Vitamin B12-Mangel und Osteoporose) [2].

EIM sind bei IBD Patienten häufig. Prävalenzen von 6-47\% werden in der Literatur beschrieben [3-5]. In der Schweizer IBD Kohorte (Swiss IBD cohort study, SIBDC) sind 29\% aller Patienten von EIM betroffen (Abbildung 1) [3]. Die Mehrheit der Patienten berichtet von einer EIM (63\%), häufig treten jedoch auch 2, 3, 4 oder 5 EIM gleichzeitig oder nacheinander auf $(27 \%, 5 \%, 2.5 \%, 2.5 \%)$.

Die Symptomatik der EIM muss dabei nicht parallel zur Entzündungsaktivität im Darm verlaufen. Bis zu 25\% aller EIM werden bereits vor der IBD Diagnose festgestellt (Median: 5 Monate, in Extremfällen bis zu 20 Jahre). Typischerweise treten EIM jedoch gleichzeitig oder nach der IBD Diagnose auf (Verzögerung im Median 8 Jahre, maximal 40 Jahre). Insgesamt kumuliert während des Krankheitsverlaufes die Wahrscheinlichkeit einer EIM und $50 \%$ aller IBD Patienten sind 30 Jahre nach Erstdiagnose von mindestens einer EIM betroffen [3].

\section{Klassifikation der Gelenkbeschwerden bei IBD}

Gelenkbeschwerden sind die häufigsten extraintestinalen Beschwerden in der Schweizer IBD-Kohorten Studie (SIBDCS): 49\% aller EIM betreffen periphere Gelenke und 11\% das Achsenskelett (Abbildung 2) [3].

Gelenkbeschwerden bei IBD umfassen nicht-entzündliche Arthralgien und entzündliche Spondylarthritiden [2]. Arthralgien und Spondylarthritiden können das Achsenskelett und die peripheren Gelenke betreffen. Die Unterscheidung von den insgesamt häufigeren Arthralgien (nicht-entzündlicher Gelenkbefall) von Arthritiden (entzündlicher Gelenkbefall) sollte im Zweifelsfall durch den Rheumatologen erfolgen und nur Arthritiden sind als 
periphere Spondylarthropathien aufzufassen. Für die Diagnose einer peripheren Spondyloarthritis muss ein entzündetes Gelenk mit typischen klinischen Befunden (Rötung, Erwärmung, Gelenkerguss) vorliegen. Die axiale Spondyloarthritis manifestiert sich häufig mit einem entzündlichen Rückenschmerz (s. unten) und einem typischen Befund im MRI oder Röntgenbild.

Differentialdiagnostisch muss bei Monarthritis (isolierte Entzündung eines einzelnen Gelenkes) auch an eine septische Arthritis gedacht werden (vor allem unter Immunsuppression), weiterhin kann eine Fraktur oder eine Osteonekrose bestehen (vor allem nach Steroidgebrauch). Arthralgien können auch durch degenerative Gelenkveränderungen bedingt sein oder selten Nebenwirkungen von IBD-Medikamenten wie Tumornekrosefaktor (TNF)-Inhibitoren oder Azathioprin entsprechen [2].

In der SIBDCS wurden nicht-entzündliche Gelenkschmerzen (Arthralgien) nicht konsequent von entzündlichen Gelenkschmerzen (Spondylarthritiden) unterschieden und der Anteil Patienten mit Arthritiden und peripheren Spondylarthropathien dürfte deutlich niedriger sein.

\section{Pathogenese der EIM}

Die Pathogenese von EIM ist bis heute nur unvollständig verstanden. Eine genetisches Risiko für EIM ist wahrscheinlich, denn bei Verwandten mit IBD ist eine Konkordanz der betroffenen Familienmitglieder bezüglich EIM überzufällig häufig (70\% Konkordanz bei Eltern-Kind-Paaren und $84 \%$ bei Geschwisterpaaren) [6]. Als genetische Faktoren wurden humane Leukozytenantigene-(HLA-Moleküle) identifiziert. Axiale Spondylarthritiden sind mit HLA-B27 assoziiert. Bei ankylosierender Spondylitis (Morbus Bechterew) haben 94\% der Betroffenen HLA-B27, bei axialer Spondyloarthritis bei IBD liegt der Anteil bei $70 \%$. Aufgrund vieler negativer Fälle und wegen der hohen Prävalenz von bis zu 10\% in der gesunden europäischen Bevölkerung ist HLA-B27 als diagnostischer Test bei IBD Patienten ungeeignet [2]. HLA-B27 Trägerschaft erhöht bei sonst gesunden Personen das Risiko einer axialen Spondyloarthritis um den Faktor 52 [7-9].

Es wurde postuliert, dass axiale Spondylarthritiden und andere EIM durch eine Kreuzreaktivität von intestinalen und/ oder mikrobiellen Antigenen mit extraintestinalen Antigenen (molekulares Mimikry) entstehen könnten. Für diese Hypothese gibt es jedoch bislang keine direkte experimentelle Evidenz. Alternative Erklärungsversuche basieren auf einem 'Misfolding' des HLA-B27-Moleküls mit konsekutiver Aktivierung entzündlicher Prozesse via IL-23R ${ }^{+}$Zellen [10]. 
Interessanterweise ist das Vorliegen eines HLA-B27 Genotyps nicht zwingend mit einer Erkrankung verbunden und für die Abwehr gegen einige Pathogene ein Vorteil: Die immunologische Aufgabe von HLA-Molekülen ist die Präsentation von Peptidmolekülen an T-Zellen und HLA-B27 kann Peptide von Influenza-Viren und Chlamydien besonders effizient binden [11]. HLA-B27 wurde ausserdem mit einem günstigen klinischen Verlauf bei Patienten mit humanem Immundefizienz-Virus (HIV) („long-term non progression“) assoziiert [12]. HLA-B27 scheint also ein hocheffizientes Verteidigungsmolekül zu sein, mit dem Preis des erhöhten Risikos für Autoimmunerkrankungen.

\section{Axiale Spondyloarthritis}

Axiale Spondyloarthritis bei IBD gehört zur Krankheitsgruppe der Spondylarthritiden (SpA), entzündlichen Gelenkerkrankungen mit überlappenden Charakteristika. Die klassische Manifestation Spondylitis ankylosans (wörtlich: „immobilisierende Entzündung der Wirbelkörper") wird Morbus Bechterew genannt. Andere SpA sind die juvenile Spondyloarthritis, die reaktive Arthritis (z.B. nach bakterieller intestinaler Infektion, früher: M. Reiter), die Psoriasisarthritis oder die Spondyloarthritis bei IBD. Alle SpA sind assoziiert mit HLA-B27 und Rheumafaktor negativ. Interessanterweise ist auch der Morbus Bechterew mit Darmerkrankungen assoziiert, so entwickeln 5-10\% aller BechterewPatienten eine IBD im Verlauf und 70\% zeigen bei genauer Untersuchung eine subklinische Darmentzündung [7].

Sakroiliitis ist bei IBD häufig und betrifft $20-50 \%$ aller Patienten [2]. Die meisten dieser Patienten sind jedoch asymptomatisch, mit geringem Risiko für Krankheitsprogression (s.u.) und müssen nicht behandelt werden. Eine beidseitige Sakroiiliitis erhöht jedoch das Risiko einer Progression [13].

Typische entzündliche Rückenschmerzen werden von 5-30\% aller IBD Patienten berichtet [13]. Für die Diagnose sind die Beschwerden des Patienten wegweisend: Für entzündliche Rückenschmerzen müssen 4 von 5 der folgenden Kriterien erfüllt sein: Erkrankungsbeginn <40 Jahre, schleichender Beginn, Dauer $\geq 3$ Monate, Morgensteifigkeit und Besserung nach Bewegung [14]. Bei Patienten, die die Kriterien für entzündliche Rückenschmerzen erfüllen, sollte unmittelbar ein MRI von Wirbelsäule und lleosakralgelenk durchgeführt werden um entzündliche Veränderungen (Sacroiliitis, Spondylitis, Spondylodiszitis, Arthritis von Costovertebral- und Facettengelenken, Enthesitis) und strukturelle Läsionen (fettige Degeneration, Erosionen, subchondrale Sklerosen, Syndesmophyten, Ankylose) nachzuweisen, die für die Gelenkfunktion prognostisch 
relevant sind [2]. Konventionelle Röntgenbilder von Wirbelsäule und Beckens zeigen vor allem Spätmanifestationen der Erkrankung.

Eine Krankheitsprogression mit Gelenkdestruktion und dem Risiko der Wirbelsäulenversteifung findet sich bei 1-10\% aller IBD Patienten [2]. Radiologisch kommt es zu Erosionen und Sklerosierungen am Gelenkspalt und in schwereren Fällen zu einer Aufhebung und Versteifung des lleosakralgelenkes (ISG) und der Wirbelsäulengelenke. An den Wirbelkörpern bilden sich Fortsätze („Syndesmophyten“), die mehrere Wirbelkörper verbinden („bridging syndesmophytes“) und letztendlich im Sinne einer „Bamboo-spine“ die Wirbelsäule immobilisieren können.

SpA haben typische extraspinale Manifestationen. So kann es zur Enthesitis, einer schmerzhaften Entzündung der Sehnenansätze (Enthesen) kommen. Prädilektionsstellen sind der Calcaneus mit den Ansätzen für die Achillessehne und die Plantarfaszie.

Eine Daktylitis ist die Entzündung eines gesamten Fingers oder einer gesamten Zehe im Strahl („sausage-finger“ oder „sausage-toe“). Eine Daktylitis ist ein relativ spezifisches Zeichen einer zugrunde liegenden Spondyloarthritis [13], betrifft jedoch nur 2-4\% aller IBD Patienten [2].

\section{Periphere Spondylarthritiden bei IBD}

Bei peripheren Spondylarthritiden wird von Gastroenterologen empirisch Gelenkbefall Typ 1 nach Orchard mit Befall von $<5$ meist grossen Gelenken insbesondere der unteren Extremitäten (Kniegelenk) vom Typ 2 nach Orchard mit Befall von $\geq 5$ Gelenken meist der Hände (Metacarpophalangealgelenk) unterschieden [15]. Der Gelenkbefall ist beim Typ 1 typischerweise nie, bei Typ 2 kaum destruierend, die Bildgebung peripherer Gelenke ist daher bei IBD-Patienten häufig unergiebig.

Diese Unterscheidung erscheint aus gastroenterologischer Sicht sinnvoll denn beim Typ 1 treten die Beschwerden akut, entzündungsbegleitend während des Krankheitsschubes einer IBD auf und sind nach 10 Wochen, mit Besserung der Darmentzündung praktisch immer verschwunden. Beim Typ 2 sind die Gelenkschmerzen unabhängig von der Krankheitsaktivität des Darmes und meist über Monate oder Jahre anhaltend. Orchard Typ 1 und 2 werden in der rheumatologischen Literatur jedoch nicht unterschieden und es bleibt offen, ob bei einer rigorosen rheumatologischen Untersuchung mit konsequentem Ausschluss simpler Arthralgien diese Unterteilung aufrechterhalten werden könnte. 


\section{Therapie der Spondylarthritiden}

Für Spondylarthritiden bei IBD sind mehrere Behandlungsmöglichkeiten etabliert (Tabelle 2) [2]. Für Spondylarthritiden gibt es exzellente Daten aus rheumatologischen Studien. Da Spondylarthritiden eine Familie ähnlicher Krankheitsbilder darstellen, scheinen diese Behandlungsergebnisse mit wenigen Einschränkungen (s. unten) auf IBD Patienten übertragbar zu sein. Die Therapie sollte, wenn irgend möglich interdisziplinär gestaltet werden, mit guter Zusammenarbeit von Gastroenterologen und Rheumatologen unter Einbeziehung des Patienten [16].

Therapeutisch werden bei axialen Spondylarthritiden medikamentöse und nichtmedikamentöse Therapien unterschieden. Der Patient sollte zur Nikotinkarenz aufgefordert werden, da Rauchen Entzündungsaktivität und Syndesmophytenbildung fördern kann [17, 18]. Leider ist Nikotinkonsum vor allem bei IBD Patienten mit Morbus Crohn häufig [19] und intensiver Zigarettenkonsum kann bei Morbus Crohn die Krankheit verschlimmern [20].

Die Effektivität einer Physiotherapie wurde in einer Cochrane Metanalyse gezeigt [21]. Dabei ist ein supervisiertes Gruppentraining häuslichen Übungen nach vorheriger Instruktion überlegen. Die beste Therapieoption scheint ein intensives Training über mehrere Wochen, idealerweise in Verbindung mit Heilbädern oder Hydrotherapie gefolgt von supervisierter Physiotherapie zu sein. Kuren bzw. Physiotherapie sind resourcenintensiv, angesichts des signifikanten Nutzens jedoch insgesamt kosteneffizient [22].

Die Wirksamkeit von nicht-steroidalen Antirheumatika (NSAR) ist bei axialen Spondylarthritiden sehr gut belegt. Bei IBD-Patienten ist jedoch vor allem bei klassischen nicht-selektiven NSAR (z.B. Ibuprofen, Naproxen, Diclofenac) Vorsicht geboten, da insbesondere in hohen Dosen Schübe einer Darmentzündung auftreten können [2, 23]. Daher sollten Cox-2 Hemmer (z.B. Celecoxib oder Etoricoxib) [24] oder enteral als relativ sicher einzustufende NSAR mit relativer Cox-2-Selektivität (z.B. Etodolac) oder andere Schmerzmedikamente (z.B. Paracetamol, Metamizol, Tramadol) verwendet werden.

Eine Metaanalyse mit 4363 Patienten zeigte bei Spondyloarthritispatienten positive Wirkungen von Cox-2-Inhibitoren auf Schmerzen, Krankheitsaktivität und Gelenkfunktion bei nur wenigen Nebenwirkungen (24/1000 Individuen bei vs. 11/1000 bei Placebo) [25]. Sollten Bewegung/ Physiotherapie sowie NSAR/ Cox-2-Hemmer nicht ausreichen ( $\geq 4$ Wochen erfolglose konservative Therapie), kann die Therapie auf einen TNF-Hemmer eskaliert werden. Bei Behandlung aus rein rheumatologischer Indikation ist die Beurteilung durch einen Rheumatologen zur Diagnosesicherung und zur Abschätzung der 
Krankheitsaktivität (BASDAl: Bath Ankylosing Spondylitis Disease Activity Index oder ASDAS: Ankylosing Spondylitis Disease Activity Score) empfohlen [16]. Therapeutisch können Infliximab, Adalimumab, Golimumab oder Certolizumab angewendet werden. Etanercept sollte bei fehlenden therapeutischen Effekten auf Darmentzündung bei IBD Patienten nicht angewendet werden. TNF-Hemmer sind trotz höherer Therapiekosten Medikamente der Wahl bei refraktären axialen Spondylarthritiden [2] und eine Vielzahl von rheumatologischen Studien belegt die Effizienz von TNF-Hemmern zur Sicherung der Gelenkfunktion [26]. Kürzlich konnte gezeigt werden, dass TNF-Hemmer auch die radiologische Progression einer ankylosierenden Spondylitis verhindern können [27]. Neue Daten von der Schweizer IBD Kohorte haben ebenfalls die Wirksamkeit von TNFInhibitoren bei EIM aufgezeigt [28, 29].

Kleinere Studien legen nahe, dass diese Ergebnisse auch auf Patienten mit IBD und axialen Spondylarthritiden übertragbar sind [30]. In der Schweizer IBD-Kohortenstudie sind von 366 Patienten mit EIM 213 mit einem TNF-Inhibitor behandelt worden. Unter Infliximab bzw. Adalimumab war die Therapie bei $74 \%$ bzw. $70 \%$ der Patienten erfolgreich gewesen [28].

Bei peripherer Spondyloarthritis steht eine Schmerztherapie (wenn irgend möglich ohne NSAR) im Vordergrund, sowie ein Therapieversuch mit Sulfasalazin. Sulfasalazin hat ebenfalls einen positiven Effekt auf die IBD. Methotrexat stellt ein alternatives Medikament zur Behandlung von peripheren Arthritiden bei IBD dar, wobei ebenfalls ein Effekt auf die IBD erwartet werden kann. Steroide können bei oligoartikulärem Befall lokal in das betroffene Gelenk injiziert oder kurzzeitig oral gegeben werden. Bei Behandlung von Gelenkbefall Typ 1 nach Orchard bessert oft eine Behandlung der Grundkrankheit die Gelenkbeschwerden. Nach $\geq 4$ Wochen erfolgloser Therapie kann nach rheumatologischer Beurteilung eine Therapie mit einem TNF-Hemmer erwogen werden [2, 16]. Bei Arthralgien steht die symptomatische Behandlung der Schmerzen im Vordergrund.

\section{Osteoporose und Osteopenie bei IBD}

Bei IBD Patienten sind Demineralisierungen der Knochen sehr häufig. Eine erniedrigte Knochendichte (Osteopenie) besteht bei $35-75 \%$ aller IBD Patienten, eine bedrohlich niedrige Knochendichte (Osteoporose) bei 10-40\% [31-33]. Das Frakturrisiko ist bei IBD Patienten gegenüber Kontrollpersonen insgesamt um etwa 50\% erhöht [34, 35].

Die Knochendichte wird mittels Dual-Energy X-Ray Absorptiometry (DXA) gemessen und mit einer gesunden Referenzpopulation verglichen. Dabei gibt der T-Score die Abweichung zu einer gesunden (25-30-jährigen) Referenzpopulation desselben Geschlechts an, 
während für den Z-Score bei der Referenzpopulation zusätzlich Alter und Ethnie berücksichtigt wird. Der Z-Score sollte für prämenopausale Frauen und Männer vor dem 50. Lebensjahr benutzt werden. Eine altersbezogen erniedrigte Knochendichte besteht bei einem Z-Score $\leq-2.0$. Der T-Score wird für Männer über 50 Jahre und peri-, bzw. postmenopausale Frauen angegeben und in «normal» (T-Score > -1.0), «Osteopenie» (TScore -1.1 bis -2.4) und «Osteoporose» (T-Score < -2.5) eingeteilt. Eine Verminderung der Knochendichte um eine Standardabweichung führt etwa zu einer Verdoppelung des Frakturrisikos.

\section{Pathogenese verminderter Knochendichte bei IBD}

Der negative Einfluss der IBD auf Knochendichte und -qualität ist multifaktoriell. So kann die chronische Entzündung eine vermehrte Knochenresorption zur Folge haben. Weiterhin kann eine Dünndarmdysfunktion oder chronische Diarrhoe zu einer Malabsorption führen. Bei intestinaler Entzündung besteht zudem oft eine sekundäre Laktoseintoleranz, die zur Milch- und damit kalziumarmen Ernährung führt. Als weiterer Faktor kann eine längerdauernde Steroidtherapie sowohl Knochendichte vermindern als auch zusätzlich und unabhängig davon die Knochenmikroarchitektur schädigen. Übereinstimmend mit diesen Risikofaktoren konnte eine Osteoporose bei IBD Patienten insbesondere bei Morbus Crohn, höherem Alter, niedrigem Body Mass Index, hoher Krankheitsaktivität, Steroidgebrauch, perianaler Erkrankung und Malabsorption gefunden werden [36].

\section{Diagnostik}

Bei IBD-Patienten mit hohem Risiko für Osteopenie oder Osteoporose ist bei Osteoporosescreening empfohlen. Die Indikation für ein Screening wird von verschiedenen gastroenterologischen Leitlinien jedoch unterschiedlich definiert (Tabelle 3). Ein längerer Steroidgebrauch ist eine anerkannte Indikation für ein Osteoporosescreening. Englische und amerikanische Leitlinien erwähnen zudem eine persistierend aktive Darmentzündung [37-40]. Der kumulative Steroidgebrauch eines IBD Patienten ist jedoch in der Praxis nur schwer zu rekonstruieren. Genauso ist in Abwesenheit klarer Schwellenwerte für eine „persistierend aktive“ Darmentzündung dieses Kriterium unscharf. Neueste Empfehlungen der European Crohn's and Colitis Organization (ECCO) unterscheiden daher bzgl. Osteoporosescreening nicht zwischen Patienten mit und ohne IBD [2].

Osteoporosescreening wird in der Praxis bei IBD Patienten unterschiedlich gehandhabt. So unterschieden sich die Screening Raten in 6 grossen Schweizer IBD-Behandlungszentren markant $(20 \%-72 \%)$, diese Unterschiede blieben auch nach Kontrolle für Risikofaktoren 
für Osteoporose erhalten [41]. Die Entscheidung für ein Osteoporosescreening wird deshalb offenbar oft individuell gefällt.

\section{Therapie von Osteopenie und Osteoporose}

Vitamin D Spiegel sollten bei allen IBD-Patienten mindestens jährlich bestimmt werden. Ein Vitamin D Mangel sollte in jedem Fall behandelt werden, wobei die untere Grenze für die Substitution umstritten ist (20 oder $30 \mathrm{ng} / \mathrm{ml}$ ) [42]. Neben positiven Effekten auf die Knochendichte wird eine immunmodulatorische entzündungshemmende Wirkung von Vitamin $D$ bei Darmentzündungen angenommen, die bei IBD Patienten therapeutisch sinnvoll wäre [2]. Da Vitamin D als fettlösliches Vitamin gut gespeichert wird, kann die Substitution auch in grösseren Abständen erfolgen (z.B. 40'000 IU einmalig am Monatsanfang), um die Compliance des Patienten zu verbessern.

Bei allen Patienten sollte zudem die tägliche Kalziumzufuhr erfragt werden (z.B. mittels standardisierter Fragebögen, von der Rheumaliga hier herunterzuladen: https://www.rheumaliga.ch/assets/doc/CH Dokumente/divers/Calciumrechner mit-

Logo.pdf). Sollte der Patient weniger als $1000 \mathrm{mg}$ Kalzium pro Tag zu sich nehmen wäre eine Substitution indiziert. Die ECCO-Leitlinien empfehlen zudem eine Substitution von $1000 \mathrm{mg}$ Kalzium und $\geq 1000$ IU Vitamin D pro Tag bei Osteopenie mit einem T-Score $\leq-1.5$ und während anhaltender Steroidmedikation [2].

Bei tiefer Knochendichte oder Frakturen und/ oder weiteren Risikofaktoren (z.B. Frakturen, familiäre Belastung mit Osteoporose oder Rauchen) muss zudem eine geeignete spezifische Osteoporose-Therapie mit dem Patienten besprochen und eingeleitet werden. Möglichkeiten sind perorales Alendronat oder die parenterale Gabe von Ibandronat oder Zoledronat. Osteoporosemedikamente sind dabei jedoch für jüngere IBD Patienten nicht getestet worden und die Therapie bleibt immer eine Einzelfallentscheidung. Vor Antiresorptiver Behandlung muss auf einen ausgeglichenen Kalzium- und Vitamin D Spiegel geachtet werden. Bei persistierend aktiver Erkrankung und grenzwertigen T-Scores können die Osteoporosemessungen auch jährlich wiederholt werden [2].

\section{Korrespondenzadresse}

PD Dr. med. Benjamin Misselwitz

Klinik für Viszerale Chirurgie und Medizin, Bauchzentrum

Freiburgstrasse 18, 3010 Bern

benjamin.misselwitz@, insel.ch 


\section{Bibliografie}

1. Fagagnini, S., et al., Risk factors for gallstones and kidney stones in a cohort of patients with inflammatory bowel diseases. PLoS One, 2017. 12(10): p. e0185193.

2. Harbord, M., et al., The First European Evidence-based Consensus on Extra-intestinal Manifestations in Inflammatory Bowel Disease. J Crohns Colitis, 2016. 10(3): p. 23954.

3. Vavricka, S.R., et al., Extraintestinal Manifestations of Inflammatory Bowel Disease. Inflamm Bowel Dis, 2015. 21(8): p. 1982-92.

4. Lakatos, L., et al., Association of extraintestinal manifestations of inflammatory bowel disease in a province of western Hungary with disease phenotype: results of a 25-year follow-up study. World J Gastroenterol, 2003. 9(10): p. 2300-7.

5. Veloso, F.T., Extraintestinal manifestations of inflammatory bowel disease: do they influence treatment and outcome? World J Gastroenterol, 2011. 17(22): p. 2702-7.

6. Satsangi, J., et al., Clinical patterns of familial inflammatory bowel disease. Gut, 1996. 38(5): p. 738-41.

7. Brown, M.A., T. Kenna, and B.P. Wordsworth, Genetics of ankylosing spondylitis-insights into pathogenesis. Nat Rev Rheumatol, 2016. 12(2): p. 81-91.

8. Wordsworth, P., Arthritis and inflammatory bowel disease. Curr Rheumatol Rep, 2000. 2(2): p. 87-8.

9. Mahmoudi, M., et al., New insights toward the pathogenesis of ankylosing spondylitis; genetic variations and epigenetic modifications. Mod Rheumatol, 2016: p. 1-12.

10. Smith, J.A., Regulation of Cytokine Production by the Unfolded Protein Response; Implications for Infection and Autoimmunity. Front Immunol, 2018. 9: p. 422.

11. Bowness, P., HLA B27 in health and disease: a double-edged sword? Rheumatology (Oxford), 2002. 41(8): p. 857-68.

12. Carrington, M. and S.J. O'Brien, The influence of HLA genotype on AIDS. Annu Rev Med, 2003. 54: p. 535-51.

13. Brakenhoff, L.K., et al., The joint-gut axis in inflammatory bowel diseases. J Crohns Colitis, 2010. 4(3): p. 257-68.

14. Calin, A., et al., Clinical history as a screening test for ankylosing spondylitis. JAMA, 1977. 237(24): p. 2613-4.

15. Orchard, T.R., B.P. Wordsworth, and D.P. Jewell, Peripheral arthropathies in inflammatory bowel disease: their articular distribution and natural history. Gut, 1998. 42(3): p. 387-91.

16. van der Heijde, D., et al., 2016 update of the ASAS-EULAR management recommendations for axial spondyloarthritis. Ann Rheum Dis, 2017. 76(6): p. 978991.

17. Poddubnyy, D., et al., Baseline radiographic damage, elevated acute-phase reactant levels, and cigarette smoking status predict spinal radiographic progression in early axial spondylarthritis. Arthritis Rheum, 2012. 64(5): p. 1388-98.

18. Chung, H.Y., et al., Smokers in early axial spondyloarthritis have earlier disease onset, more disease activity, inflammation and damage, and poorer function and health-related quality of life: results from the DESIR cohort. Ann Rheum Dis, 2012. 71(6): p. 809-16. 
19. Biedermann, L., et al., High Rates of Smoking Especially in Female Crohn's Disease Patients and Low Use of Supportive Measures to Achieve Smoking Cessation--Data from the Swiss IBD Cohort Study. J Crohns Colitis, 2015. 9(10): p. 819-29.

20. Lang, B.M., et al., Genetic polymorphisms associated with smoking behaviour predict the risk of surgery in patients with Crohn's disease. Aliment Pharmacol Ther, 2018. 47(1): p. 55-66.

21. Dagfinrud, H., T.K. Kvien, and K.B. Hagen, Physiotherapy interventions for ankylosing spondylitis. Cochrane Database Syst Rev, 2008(1): p. CD002822.

22. Van Tubergen, A., et al., Cost effectiveness of combined spa-exercise therapy in ankylosing spondylitis: a randomized controlled trial. Arthritis Rheum, 2002. 47(5): p. 459-67.

23. Takeuchi, K., et al., Endogenous prostaglandin E2 accelerates healing of indomethacin-induced small intestinal lesions through upregulation of vascular endothelial growth factor expression by activation of EP4 receptors. J Gastroenterol Hepatol, 2010. 25 Suppl 1: p. S67-74.

24. Sandborn, W.J., et al., Safety of celecoxib in patients with ulcerative colitis in remission: a randomized, placebo-controlled, pilot study. Clin Gastroenterol Hepatol, 2006. 4(2): p. 203-11.

25. Kroon, F.P., et al., Non-steroidal anti-inflammatory drugs (NSAIDs) for axial spondyloarthritis (ankylosing spondylitis and non-radiographic axial spondyloarthritis). Cochrane Database Syst Rev, 2015(7): p. CD010952.

26. Callhoff, J., et al., Efficacy of TNFalpha blockers in patients with ankylosing spondylitis and non-radiographic axial spondyloarthritis: a meta-analysis. Ann Rheum Dis, 2015. 74(6): p. 1241-8.

27. Molnar, C., et al., TNF blockers inhibit spinal radiographic progression in ankylosing spondylitis by reducing disease activity: results from the Swiss Clinical Quality Management cohort. Ann Rheum Dis, 2018. 77(1): p. 63-69.

28. Vavricka, S.R., et al., Anti-TNF Treatment for Extraintestinal Manifestations of Inflammatory Bowel Disease in the Swiss IBD Cohort Study. Inflamm Bowel Dis, 2017. 23(7): p. 1174-1181.

29. Greuter, T., et al., Extraintestinal Manifestations of Pediatric Inflammatory Bowel Disease: Prevalence, Presentation, and Anti-TNF Treatment. J Pediatr Gastroenterol Nutr, 2017. 65(2): p. 200-206.

30. Generini, S., et al., Infliximab in spondyloarthropathy associated with Crohn's disease: an open study on the efficacy of inducing and maintaining remission of musculoskeletal and gut manifestations. Ann Rheum Dis, 2004. 63(12): p. 1664-9.

31. Larsen, S., K. Bendtzen, and O.H. Nielsen, Extraintestinal manifestations of inflammatory bowel disease: epidemiology, diagnosis, and management. Ann Med, 2010. 42(2): p. 97-114.

32. Levine, J.S. and R. Burakoff, Extraintestinal manifestations of inflammatory bowel disease. Gastroenterol Hepatol (N Y), 2011. 7(4): p. 235-41.

33. Rothfuss, K.S., E.F. Stange, and K.R. Herrlinger, Extraintestinal manifestations and complications in inflammatory bowel diseases. World J Gastroenterol, 2006. 12(30): p. 4819-31.

34. Bernstein, C.N., et al., The incidence of fracture among patients with inflammatory bowel disease. A population-based cohort study. Ann Intern Med, 2000. 133(10): p. 795-9. 
35. Card, T., et al., Hip fractures in patients with inflammatory bowel disease and their relationship to corticosteroid use: a population based cohort study. Gut, 2004. 53(2): p. 251-5.

36. Schule, S., et al., Prediction of low bone mineral density in patients with inflammatory bowel diseases. United European Gastroenterol J, 2016. 4(5): p. 669676.

37. N.R., L. and B.B. Scott. Guidelines for osteoporosis in inflammatory bowel disease and coeliac disease. 2007 [cited 2016 26.10.]; Available from: http://www.bsg.org.uk/pdf word docs/ost coe ibd.pdf.

38. Lichtenstein, G.R., et al., Management of Crohn's disease in adults. Am J Gastroenterol, 2009. 104(2): p. 465-83; quiz 464, 484.

39. Kornbluth, A., D.B. Sachar, and G. Practice Parameters Committee of the American College of, Ulcerative colitis practice guidelines in adults: American College of Gastroenterology, Practice Parameters Committee. Am J Gastroenterol, 2010. 105(3): p. 501-23; quiz 524.

40. Bernstein, C.N., W.D. Leslie, and M.S. Leboff, AGA technical review on osteoporosis in gastrointestinal diseases. Gastroenterology, 2003. 124(3): p. 795-841.

41. Schüle, S., et al., Widely differing screening and treatment practice for osteoporosis in patients with inflammatory bowel diseases in the Swiss IBD Cohort study. Medicine, 2017.

42. Bischoff-Ferrari, H.A., Optimal serum 25-hydroxyvitamin D levels for multiple health outcomes. Adv Exp Med Biol, 2014. 810: p. 500-25. 
Tabellen

\begin{tabular}{|c|c|}
\hline $\begin{array}{l}\text { Extraintestinale Manifestationen } \\
\text { "Entzündung, nicht im Darm" }\end{array}$ & $\begin{array}{l}\text { Extraintestinale Komplikationen } \\
\text { "Folge der Entzündung" }\end{array}$ \\
\hline - Axiale Spondyloarthritis & - Osteoporose \\
\hline - Periphere Spondyloarthritis & - $\quad$ Malabsorption \\
\hline - $\quad$ Erythema nodosum & - $\quad$ Nierensteine \\
\hline - $\quad$ Pyoderma gangränosum & - Gallensteine \\
\hline - $\quad$ Sweet's Syndrom & - $\quad$ Periphere Neuropathie \\
\hline - Aphthöse Stomatitis & - $\quad$ Thrombosen \\
\hline - $\quad$ Primär sklerosierende Cholangitis & \\
\hline - Uveitis, Episkleritis & \\
\hline
\end{tabular}

Tabelle 1: Extraintestinale Manifestationen und Komplikationen bei IBD 


\begin{tabular}{|c|c|}
\hline Axiale Spondyloarthritis & Periphere Spondyloarthritis \\
\hline Cox-2-Inhibitoren, evtl. kurzzeitig NSAR*, & Cox-2-Inhibitoren, evtl. kurzzeitig NSAR*, \\
\hline $\begin{array}{ll}\text { Physiotherapie } \\
\text { - Supervisierte Physiotherapie in } \\
\text { Gruppen } \\
\text { • Hydrotherapie }\end{array}$ & Intraartikuläre oder orale Steroide \\
\hline Kontakt zu Selbsthilfegruppen vermitteln & Sulfasalazin \\
\hline \multirow[t]{3}{*}{ TNF-Inhibitor ${ }^{* \star} z$} & Methotrexat \\
\hline & Behandlung der Grundkrankheit \\
\hline & $\begin{array}{l}\text { TNF-Inhibitor (Zweitlinientherapie } \\
\text { bei refraktären Beschwerden**. }\end{array}$ \\
\hline enz für alle Patienten & \\
\hline
\end{tabular}

Tabelle 2: Behandlungsmöglichkeiten bei Spondylarthritiden bei IBD.

*: Längere Therapiedauer in höheren Dosen sollte vermieden werden da ein Schub einer IBD ausgelöst werden kann, Cox-2-selektivere NSAR sollten bevorzugt werden (z.B. Etodolac)

**: Rücksprache mit Rheumatologen wenn Gabe aus alleiniger rheumatologischer Indikation geplant 


\begin{tabular}{|l|l|l|}
\hline $\begin{array}{l}\text { British Society of } \\
\text { Gastroenterology } \\
\text { (BSG, 2007) [37] }\end{array}$ & $\begin{array}{l}\text { European Crohn's and } \\
\text { Colitis Organization } \\
\text { (ECCO) [2] }\end{array}$ & $\begin{array}{l}\text { American College of } \\
\text { Gastroenterology (ACG) } \\
\text { 2009 (CD) [38], 2010 (UC) } \\
\text { [39], American } \\
\text { Gastroenterological } \\
\text { Association (AGA) 2003 [40] }\end{array}$ \\
\hline $\begin{array}{l}\geq 7.5 \text { mg/d für } \geq 6 \text { Monate } \\
\text { Risikofaktoren }\end{array}$ & $\begin{array}{l}\text { Kumulative } \\
\text { Steroidexposition über } \\
\text { Persistierende/anhaltende/ }\end{array}$ & $\begin{array}{l}\text { Kumulative Steroidexposition } \\
\text { über >3 Monate }\end{array}$ \\
\hline aktive Erkrankung & & Penate \\
\hline
\end{tabular}

Tabelle 3: Indikationen für ein Osteoporosescreening gemäss verschiedener Leitlinien

\section{Legenden zu den Abbildungen}

Abbildung 1: Häufigkeit von EIM in der Schweizer IBD Kohorte [3]

Abbildung 2: Verteilung von EIM in der Schweizer IBD Kohorte [3] 